\title{
Autism spectrum disorder in patients with inherited metabolic disorders-a large sample from a tertiary center
}

\author{
Tuba Çelen Yoldaş ${ }^{1 \oplus}$, Berrak Bilginer Gürbüz ${ }^{2 \oplus}$, Halil Tuna Akar ${ }^{2 \oplus}$, \\ Elif Nursel Özmert ${ }^{1 \oplus}$, Turgay Coşkun ${ }^{2 \oplus}$ \\ Divisions of ${ }^{1}$ Developmental Pediatrics, ${ }^{2}$ Pediatric Metabolism, Department of Pediatrics, Hacettepe University Faculty of Medicine, \\ Ankara, Turkey.
}

\begin{abstract}
Background. There is increased awareness regarding the co-occurrence of autism spectrum disorder (ASD) and inherited metabolic disorders (IMD), and this is crucial for the management of both diagnoses in clinical practice. We aimed firstly to report twenty-two patients with a dual diagnosis of IMD and ASD who are still being followed up in the child metabolism outpatient clinic; secondly to evaluate the time of both IMD and ASD diagnosis and the clinical progress of their metabolic disorders to underline treatable conditions.

Methods. Among the patients admitted to the Pediatric Metabolism outpatient clinic because of IMD, twentytwo of them who had a diagnosis of ASD were included in the study. Data of the patients were collected from their medical records. The most recent progress of the patients concerning their metabolic disorder was obtained from the patients' files.

Results. Six cases with Phenylketonuria, 2 cases with partial Biotinidase Deficiency, 3 cases with Cerebral Creatine Deficiency Syndrome (CCDS), 5 cases with Mucopolysaccharidosis (MPS) Type-3b, 2 cases with MPS Type-3a, 1 case with MPS Type 4, 2 cases with Hypervalinemia and 1 case with Maple Syrup Urine Disease were all diagnosed as also having ASD. The diagnoses of CCDS and MPS Type 3 were after the diagnosis of ASD. Phenylketonuria and Mucopolysaccharidosis were the most common diagnoses in our study. In addition, rare entities such as MPS Type 3b and Type 4 and Hypervalinemia were also reported to co-occur with autism.

Conclusions. Considering the co-occurrence of both disorders and implementing intervention strategies accordingly will certainly be beneficial in clinical practice and particularly in countries with a high rate of consanguinity.
\end{abstract}

Key words: autism spectrum disorder, inherited metabolic disorders, developmental delay.

Autism spectrum disorder (ASD) is a complex neurodevelopmental disorder and the prevalence of ASD tends to be an important problem as the rate ( 1 in every 68 children) has increased. ${ }^{1}$ The heritability of ASD is quite heterogeneous and the co-occurrence of ASD with certain inherited metabolic disorders (IMD) is not rare. Furthermore the co-occurrence of both disorders is critical because the diagnosis of one may mask the other. This comorbidity

$凶$ Tuba Çelen Yoldaş

tuba.celen@hacettepe.edu.tr

Received 19th October 2020, revised 5th March 2021, 12th April 2021, accepted 3rd May 2021. is crucial for the management and progress of both diagnoses in clinical practice. ${ }^{2}$

The disruption of metabolic processes can give rise to a number of deviant effects in the central nervous system and lead to several neurodevelopmental disorders. ${ }^{2-4}$ The altered neurodevelopmental process can cause psychiatric symptoms and sometimes these symptoms can occur before irreversible neurological lesions. To prevent or decrease any disabilities associated with IMD, it is important to detect the metabolic disease as early as possible. ${ }^{3}$ As most metabolic disorders are autosomal recessive and common in countries where the rate of consanguinity is high, it is 
particularly important for those communities to decrease the degree of mortality and morbidity with early detection. 5,6 When ASD is diagnosed in children with IMD, intervention strategies including social, behavioral, cognitive therapies can be planned in addition to the treatment of such metabolic disorders., ${ }^{2,3}$ Conversely IMD should be considered in children with symptoms of ASD. Although metabolic disorders are usually recognizable with clinical signs such as dysmorphic features, ataxia, microcephaly, seizures, coma, hepatosplenomegaly and intellectual disability some of them can present by symptoms of ASD. ${ }^{7}$ A metabolic work-up should be reserved for patients bearing any clinical indicator of a metabolic disorder. ${ }^{3}$ If an IMD can be confirmed, a specific treatment may be accessible to avoid metabolic decompensation and therefore ASD may be preventable by the early treatment of IMD. ${ }^{8}$ Furthermore many of these conditions are important for implications of genetic counselling. ${ }^{3}$

As a result of the scarce data relating to the co-occurrence of the two conditions, we firstly aimed to report twenty-two patients with a dual diagnosis of IMD and ASD who are still being followed up in the child metabolism outpatient clinic. Secondly we aimed to address the time of both IMD and ASD diagnosis and the clinical progress of their metabolic disorders to underline the treatable conditions which we did not come across in the literature.

\section{Material and Methods}

The study was conducted by the Pediatric Metabolism Division and Developmental Pediatrics Division, Department of Pediatrics. The study protocol was approved by the Ethics Committee of Hacettepe University Medical Faculty (GO 18/407) and the participation involved informed consent. Among the patients admitted to the Pediatric Metabolism outpatient clinic because of IMD between March 2015 and April 2018, twenty-five of them who had a diagnosis of ASD were included in the study. The inclusion criteria of the study was having a definitive diagnosis of both IMD and ASD and exclusion criteria was to be undergoing a process of diagnosis. Each patient was examined by a child metabolism subspecialist and a developmental pediatrician and was screened for the eligibility for the study. For the final evaluation 22 patients remained who had a definitive diagnosis of both IMD and ASD. The age at diagnosis of IMD and ASD was accepted as the first time a physician defined the diagnosis. Metabolic data of the patients were collected from their medical records. Data relating to the diagnosis of ASD was acquired from the patients' files and medical records. A definite diagnosis of ASD was accepted if the patient had an official special education therapy record. Diagnosis of ASD had been given at several different child and adolescent psychiatry departments, however the treatment and follow-up of all the patients for their metabolic disorders were being continued in the same pediatric metabolism department. The clinical progress of the patients concerning their metabolic disorder was obtained from the patients' files.

\section{Data analysis}

Most of the data was defined by numbers and percentages in the study. Numerical variables were evaluated for normality and parametric tests were used for data with normal distributions whereas non-parametric tests were used otherwise. Descriptive analysis and bivariate comparisons were used to assess group differences. Chi-square was used to examine the difference in gender across the subgroups of IMD. Differences between the median diagnostic ages of the two disorders were analyzed by Wilcoxon Signed Rank. Statistical tests were considered to be significant when $p<0.05$. Statistical analyses were performed using Statistical Package for the Social Sciences 23.0.

\section{Results}

Among the 25 patients, three did not meet the inclusion criteria of the study and finally 22 
patients suffering from both disorders were enrolled in the study. In the total sample, 13 $(59.1 \%)$ were male, $9(40.9 \%)$ were female. The median ages of the patients at the time of diagnosis of IMD and ASD were 31.5 (range 0.13-108) months and 45 (range 18-100) months, respectively. There was no difference between the median diagnostic ages of the two disorders $(p=0.20)$. Across the subgroups of IMD there was no difference in gender $(\mathrm{p}=0.761)$.

The diagnoses of Cerebral Creatine Deficiency Syndrome (CCDS) and MPS Type 3a-3b were made after the diagnosis of ASD with a 52 month and 18-24 month delay, respectively. On the contrary, the diagnoses of PKU, Biotinidase Deficiency, MSUD, and MPS 4 were all diagnosed before the diagnosis of ASD. The diagnostic age of ASD and IMD were similar in patients with hypervalinemia. Detailed clinical characteristics of the patients across the subgroups are given in Table I.

Case 1 was diagnosed with PKU at 6 months of age when investigating the reason for development delay in 2005. Nationwide neonatal screening program for PKU was started in 2006 in Turkey. Her blood phenylalanine level was measured as $\mathbf{1 6 1 5 . 2}$ $\mu \mathrm{mol} / \mathrm{L}(26.92 \mathrm{mg} / \mathrm{dl})$ and she was diagnosed with classical PKU. Sequencing of the PAH gene (OMIM*612349) in the patient revealed homozygote mutation: IVS2+5G $>C$. The patient received a phenylalanine restricted diet. It was observed that the patient's compliance with diet therapy was not good. The mean serum phenylalanine value was $445.8 \mu \mathrm{mol} / \mathrm{L}$ (7.43 $\mathrm{mg} / \mathrm{dl}$ ). She was diagnosed with ASD at the age of 61 months due to her social communication deficits and self-harming behavior. At the time of diagnosis of ASD, her phenylalanine level was $840.6 \mu \mathrm{mol} / \mathrm{L}(14.01 \mathrm{mg} / \mathrm{dl})$. She was urged to comply more strictly to her diet. Seizures appeared during her clinical follow-up.

Case 2 was diagnosed with PKU at the age of 6 years because he had missed the newborn screening and he was living in institutional care. He was referred to the Pediatric Metabolism outpatient clinic because of his learning disabilities and short stature. His blood phenylalanine level was measured in our clinic and found to be $1740 \mu \mathrm{mol} / \mathrm{L}$ (29 $\mathrm{mg} / \mathrm{dl}$ ), also consistent with classical PKU. He had nonverbal communication deficits and restrictive, repetitive behaviors and, serious intellectual disability. He had received an ASD diagnosis at the age of 100 months. On his physical examination he had short stature because of growth hormone deficiency. Although phenylalanine- restricted diet therapy was implemented his behavioral difficulties did not improve.

Case 3 was referred for his developmental delay and received the diagnosis of PKU at

Table I. Characteristics of patients across subtypes of metabolic diseases.

\begin{tabular}{|c|c|c|c|c|}
\hline Metabolic Diagnosis Total sample, N=22 (\%) & $\begin{array}{c}\text { Median age of } \\
\text { IMD diagnosis } \\
\text { (month) } \\
\text { (min-max) }\end{array}$ & $\begin{array}{l}\text { Median age of } \\
\text { ASD diagnosis } \\
\text { (month) } \\
\text { (min-max) }\end{array}$ & $\begin{array}{c}\text { Gender } \\
\text { Male } \\
\mathrm{N}(\%)\end{array}$ & $\begin{array}{c}\text { Consanguinity } \\
\text { Yes } \\
\mathrm{N}(\%)\end{array}$ \\
\hline Phenylketonuria, $\mathrm{N}=6$ (27.3) & $5(0.16-72)$ & $45(36-100)$ & $3(50)$ & $1(16.7)$ \\
\hline Biotinidase deficiency, $\mathrm{N}=2$ (9.0) & $0.56(0.13-1)$ & $24(18-30)$ & $2(100)$ & 0 \\
\hline Cerebral creatine deficiency, N=3 (13.6) & $73(39-108)$ & $21(18-48)$ & $2(66.7)$ & 1(33.3) \\
\hline Mucopolysaccharidosis Type 3a, N=2 (9.0) & $72(60-84)$ & $54(48-60)$ & $1(50)$ & 0 \\
\hline Mucopolysaccharidosis Type 3b, N=5 (22.8) & $72(18-97)$ & $48(36-90)$ & $2(40)$ & 2(40.0) \\
\hline Mucopolysaccharidosis Type 4, N=1 (4.6) & 32 & 68 & 0 & $1(100)$ \\
\hline Hypervalinemia, N=2 (9.0) & $34.5(31-38)$ & $30(24-36)$ & $2(100)$ & 0 \\
\hline Maple syrup urine disease, $\mathrm{N}=1$ (4.6) & 0.26 & 84 & $1(100)$ & 0 \\
\hline
\end{tabular}

IMD: inherited metabolic disorders, ASD: autism spectrum disorder 
the age of 20 months. His blood phenylalanine level was measured as $2419.2 \mu \mathrm{mol} / \mathrm{L}(40.32$ $\mathrm{mg} / \mathrm{dl}$ ) and he was diagnosed with classical PKU. The patient was diagnosed in 2006 while investigating his developmental delay. The neonatal screening program was not active in the year when the patient was born. Phenylalanine-restricted diet therapy was implemented. The mean phenylalanine level in the follow-up of the patient was $307.2 \mu \mathrm{mol} / \mathrm{L}$ $(5.12 \mathrm{mg} / \mathrm{dl})$. He received the diagnosis of ASD at the age of 42 months due to his deficits in social communication and restricted behavior patterns. He had also intellectual disability, seizures, and hyperactivity on his follow-up.

Case 4 was referred to the Pediatric Metabolism outpatient clinic following newborn screening on postnatal day 5. Her blood phenylalanine level was measured in our clinic and found to be $925.2 \mu \mathrm{mol} / \mathrm{L}(15.42 \mathrm{mg} / \mathrm{dl})$. The patient could not be followed regularly. While she was receiving restricted diet therapy, her mean phenylalanine level was $409.2 \mu \mathrm{mol} / \mathrm{L}$ (6.82 mg/ dl). ASD was diagnosed at the age of 39 months because of her limited eye contact, stereotypical behavior, and not responding to her name. She also received special education and, her behavioral difficulties improved partially.

Case 5 was referred to the Pediatric Metabolism outpatient clinic because of jaundice and received the diagnosis of PKU at the age of 1 month. His quantitative plasma amino acid analysis was significantly high for phenylalanine at a level of $1782 \mu \mathrm{mol} / \mathrm{L}(29.7 \mathrm{mg} / \mathrm{dl})$. Sequencing of the PAH gene (OMIM*612349) in the patient revealed homozygote mutation IVS4+ $1 \mathrm{G}>\mathrm{A}$. The patient's follow-up was irregular. The mean phenylalanine level at follow-up of the patient was $333 \mu \mathrm{mol} / \mathrm{L}(5.55 \mathrm{mg} / \mathrm{dl})$. Social communication difficulties appeared at the age of 36 months and received an ASD diagnosis. After implementing diet therapy and special education behavioral symptoms improved however, his learning difficulties continued.

Case 6 was referred to the Pediatric Metabolism outpatient clinic because of suspected seizure history and developmental delay and was diagnosed with PKU at the age of 4 months in 2001.Her blood phenylalanine level was measured in our clinic and found to be 780 $\mu \mathrm{mol} / \mathrm{L}(13 \mathrm{mg} / \mathrm{dl})$. The patient's compliance with diet therapy was not good. She didn't have seizures on her follow up. Because of behavioral problems and social communication difficulties she received an ASD diagnosis at the age of 48 months. Her behavioral symptoms did not improve following diet and special education therapy. Furthermore, she received a diagnosis of the Systemic Lupus Erythematosus on her follow up.

Case 7 received the diagnosis of partial biotinidase deficiency at the age of 1 month. He was referred to the Pediatric Metabolism outpatient clinic following newborn screening. His biotinidase activity was $1.77 \mathrm{U} / \mathrm{L}(24.9 \%)$ and $1.60 \mathrm{U} / \mathrm{L}$ (22.5\%). He had c.235C> T; p.Arg79Cys heterozygous mutation. Although the mutation in the patient was heterozygous, the biotinidase activity of the patient was found to be low (checked twice), consistent with partial deficiency. His parents did not mention either hair loss or skin manifestations. Oral biotin replacement was started at a dose of 5 $\mathrm{mg}$ per day. At the age of 18 months he had an ASD diagnosis due to deficits in socioemotional reciprocity such as poorly integrated verbal and nonverbal communication. He also had seizures at follow up.

Case 8 received the diagnosis of partial biotinidase deficiency at his postnatal 4 day newborn screening. His first biotinidase activity was 3.07U/L (43.2\%), the second was $2.00 \mathrm{U} / \mathrm{L}$ (28.16\%) and, there was homozygote c.1330G>C mutation on BTD gene. His parents did not mention either hair loss or skin manifestations. The patient's hearing examination and audiogram were reported to be normal. Oral biotin replacement was started at a dose of 5 mg per day. Because of his impaired reciprocal communication and stereotypical movements, she had an ASD diagnosis at the age of 30 months. Despite continued biotin therapy and special education therapy, complete response was not achieved. 
Case 9 had an ASD diagnosis at the age of 12 months due to stereotypical hand movements and communication problems. She was referred to the metabolic outpatient clinic because of seizures, intellectual disability, and tonus hyperactivity. Serum creatinine level was 0.11 mg / dl. The level of her urine Guanidoasetat was $1414 \mu \mathrm{mol} / \mathrm{L}$. The creatinine level in spot urine was $93.91 \mathrm{mg} / \mathrm{dl}$. The cranial magnetic resonance imaging (MRI) examination of the patient was normal. In the magnetic resonance spectroscopy (MRS) examination, the creatine peak value was seen to have lost its significant level at a level close to 0 in the parietooccipital cortex. This finding was compatible with creatine deficiency syndrome. The guanidino acetate (GAA) peak was not found. Genetic test revealed c.261_269del (p.W87fsX) homozygote mutation found on guanidinoacetate methyltransferase (GAMT) gene. She received a CCDS diagnosis at the age of 108 months. She was receiving special education therapy and, despite adding the metabolic therapy with creatine monohydrate, L-ornithine and an Arginine-protein restricted diet a limited improvement was observed in her clinical findings.

Case 10 had received a diagnosis of ASD at the age of 15 months due to social communication problems. He was referred to the Pediatric Metabolism outpatient clinic because of seizures and social communication problems. His blood creatine level was $0.09 \mathrm{~g} / \mathrm{dl}$. Genetic test results were homozygous frameshift mutation, hemizygous (X linked) on SLC6A8 gene. He received the diagnosis of creatine transporter deficiency at the age of 73 months. He was receiving special education therapy. Despite adding metabolic therapy with creatine monohydrate, L-arginine, L-glycine, S-adenosyl, methionine, risperidone, and aripiprazole, a complete response was not achieved.

Case 11 was referred for global developmental delay and received a CCDS diagnosis at the age of 39 months. The level of blood creatinine was $0.28 \mathrm{mg} / \mathrm{dl}$ and blood guanidinoacetate was $0 \mu \mathrm{mol} / \mathrm{L}$. GAMT gene analysis showed a homozygous c.327G $>$ A mutation on Exon
2. Symmetrical T2 signal increase in bilateral globus pallidus was noted on the cranial MRI of the patient. In multivoxel short and intermediate echo examinations made from deep gray matter and single voxel examination involving left parietal gray-white matter composition, creatine was found to be close to 0 or 0 in all voxels. He had seizures, behavioral problems, limited self-care, and social communication difficulties. The diagnostic age of ASD was 48 months. Despite special education therapy and drug therapy such as creatine monohydrate, pregabalin, no improvement was observed in his clinical findings.

Case 12 had an ASD diagnosis at the age of 48 months. She was referred for gross motor delay and verbal and nonverbal communication and behavioral problems. On her physical examination, short stature, pectus carinatum and hepatosplenomegaly were observed. Her urine mucopolysaccharides was $15.23 \mathrm{mg} / \mathrm{dl}$ and sulphamidase activity in leucocytes was $0.13 \mathrm{nmol} / \mathrm{mg} / 17 \mathrm{hr}$ (normal range: $3.2-20.4$ ). She received a diagnosis of MPS Type3a at the age of 60 months. She received multidisciplinary supportive therapy and special education however no improvement was observed in her clinical findings.

Case 13 was diagnosed with an ASD due to delayed speech, difficulty with socialization, and restricted interest in his surroundings at the age of 60 months. He was referred to the Pediatric Metabolism outpatient clinic because of skeletal problems. His urine mucopolysaccharides was $18.12 \mathrm{mg} / \mathrm{dl}$ and he had a diagnosis of MPS Type $3 \mathrm{a}$ at the age of 84 months. He received multidisciplinary supportive therapy and special education however no improvement was observed in his clinical findings.

Case 14 was referred for seizures, global developmental delay, and physical appearance. He had a diagnosis of MPS Type3b at the age of 18 months. He had ASD diagnosis at the age of 48 months with complaints of impaired reciprocal communication and a lack of eye contact, and echolalia. He received multidisciplinary 
supportive therapy and special education however, no improvement was observed in his clinical findings.

Case 15 was referred for global developmental delay, behavioral problems and, skeletal problems, and she had a diagnosis of MPS Type $3 b$ at the age of 25 months. Plasma alpha $\mathrm{N}$ acetylglucosamidase activity was found to be severely reduced. In the genetic analysis, c.235G> T (p.G79C) homozygous mutation was detected in exon 1 in the NAGLU gene. She was diagnosed with ASD at the age of 39 months due to delayed speech and difficulty with socialization, a lack of interest in her surroundings, as well as stereotypical movements. She did not continue her follow-up regularly.

Case 16 had an ASD diagnosis at the age of 60 months because of his impaired reciprocal communication and a lack of eye contact, and echolalia. He was referred to the Pediatric Metabolism outpatient clinic because of his developmental delay and coarse facial features. A diagnosis of MPS was suspected and the leukocyte acetyl alpha glucosaminidase enzyme activity was found to be $0 \mathrm{nmol} /$ hour/ mg. He was diagnosed with MPS Type $3 b$ at the age of 72 months. Seizures were controlled by an antiepileptic. He received multidisciplinary supportive therapy and special education however no improvement was observed in his clinical findings.

Case 17 was diagnosed with an ASD due to socialization problems and a lack of interest in her surroundings, as well as stereotypical movements at the age of 90 months. She was consulted to the pediatric metabolism outpatient clinic due to atypical behavior patterns, coarse facial features, adenoid vegetation, tonsillar hypertrophy, and intellectual disability. She had a diagnosis of MPS Type $3 b$ at the age of 97 months with enzyme analyses. Despite multidisciplinary therapy, no improvement was observed in his clinical findings.
Case 18 had a diagnosis of MPS Type $3 b$ at the age of 84 months when she was referred to the metabolic outpatient clinic for short stature and intellectual disability. Acetyl-alphaglucosaminidase specific enzyme activity in plasma was found to be $0 \mathrm{nmol} / \mathrm{hour} / \mathrm{ml}$. The reference range was $5-58 \mathrm{nmol} / \mathrm{hour} / \mathrm{ml}$. Urinary glycosaminoglycan electrophoresis showed pronounced heparan sulfate band. Total urinary glucosaminoglycan level was found to be $209.3 \mathrm{mg} / \mathrm{g}$ creatinine. Healthy control values between the ages of 2-13 years were $66-371 \mathrm{mg} / \mathrm{g}$ creatinine. She had a diagnosis of ASD at the age of 36 months because of her social communication deficits and stereotypical behavior. She did not continue her follow-up regularly.

Case 19 was admitted to the general pediatric outpatient clinic with the complaint of increasing protrusion of her rib cage. After the skeletal survey, radiographical findings revealed signs of mucopolysaccharidosis and she was referred to the child metabolism department. Diagnosis of Morquio A Syndrome was made at the age of 32 months by physical examination, skeletal radiographs and keratan sulfate band in the electrophoresis of urinary GAG and nonenzymatic activity of $\mathrm{N}$ - acetylgalactosamine -6 sulfate-sulfatase. She had global developmental delay when she received the diagnosis of MPS Type 4. Enzyme therapy was started. However, she received the diagnosis of ASD at the age of 68 months because of her social communication deficits and, restricted joint attention and eye contact, echolalia, and a stereotypical head movements. Although special education therapy was implemented, her behavioral difficulties improved very little.

Case 20 was referred to the Pediatric Metabolism outpatient clinic for delayed speech, dysmorphic features, and echolalia. His combined amino acid analysis was significant for valine of 480.9 $\mathrm{nmol} / \mathrm{L}(<250 \mathrm{nmol} / \mathrm{L})$ and received a diagnosis of Hypervalinemia (valine transaminase deficiency) at the age of 31 months. Peripheral 
chromosome and FISH analyses were observed as normal. He had an ASD diagnosis at the age of 24 months because of his social communication deficits and repetitive behaviors. Following special education therapy, improvements in his behaviors were observed.

Case 21 was referred to the Pediatric Metabolism outpatient clinic for verbal and nonverbal communication problems. His combined amino acid analysis was significant for valine of 294.7 $\mathrm{nmol} / \mathrm{L}(<250 \mathrm{nmol} / \mathrm{L})$ and received a diagnosis of hypervalinemia (valine transaminase deficiency) at the age of 38 months. Further genetic investigation was not performed due to family issues. No findings were found to explain the clinical picture, except for hypervalinemia. At the age of 36 months, he was diagnosed with ASD at a similar time when IMD was detected. He had social communication deficits, restricted interests and stereotypical behaviors such as lining up toys or flipping objects. Following special education therapy, improvements in his behaviors were observed.

Case 22 was referred with restlessness, contractions in the hands and feet, decreased feeding, decreased activity and diagnosed with MSUD at his postnatal 8 days. The patient was found to have deficient leucine decarboxylation. He had $13 \mathrm{pmol} / \mathrm{hr} / \mathrm{mg} 1-{ }^{14} \mathrm{C}$ leucine decarboxylation level whereas the normal value was $784 \mathrm{pmol} / \mathrm{hr} / \mathrm{mg}$. He had verbal and nonverbal communication and selfcare problems and stereotypical behaviors. $\mathrm{He}$ was diagnosed with ASD at the age of 84 months. Restricted diet from leucine and protein was implemented however, complete response was not achieved.

Description of the clinical findings and diagnosis of the patients are presented in Table II.

\section{Discussion}

We identified twenty-two cases with inherited metabolic disorders who also had ASD.
There was no difference between the median diagnostic ages of the two disorders however, the median age of ASD diagnosis was 45 months of age. The variability in the average age of ASD diagnosis might be related to the heterogeneity of symptoms. ${ }^{9}$ Symptomatic presentation and the developmental course of ASD may differ according to whether the child has a co-occurring condition. ${ }^{10,11}$

Phenylketonuria was one of the most common diagnoses with 6 cases in the study. Consistent with previous literature, PKU is the most common genetic metabolic disorder associated withASD. ${ }^{12,13}$ Among well-documented potential pathophysiological mechanisms associated with neurodevelopmental impairment, functional and molecular alterations in the prefrontal cortex including excitation/inhibition (E/I) imbalance is noticeable in the etiology. ${ }^{12}$ Frontal and subcortical white matter changes were also reported in patients with phenylketonuria which had been treated early. ${ }^{14}$ Similarly in autism there are frontal dysfunction and connectivity problems in the cerebral white matter. Considering the prefrontal dysfunction and connectivity problems in both disorders, it is not surprising to see autism phenotype in PKU patients. ${ }^{15}$ Phenylketonuria has a prevalence of approximately 1/4,500 in Turkey. Although there is a national newborn screening program using the Guthrie test, one patient in our study was diagnosed as late as 72 months of age and his metabolic prognosis was badly controlled. The possibility of PKU should be considered in any child presenting symptoms of autism and developmental delay. This is because even late diagnosed patients may benefit from a restricted diet. $^{13}$ Further, it should be considered that having both diagnoses could make following dietary restrictions more challenging for families and patients.

Mucopolysaccharidosis type 3 (MPS 3, Sanfilippo syndrome) was another common diagnosis in the present study, 2 cases with Type-A and 5 cases with Type-B. MPS 3 is 


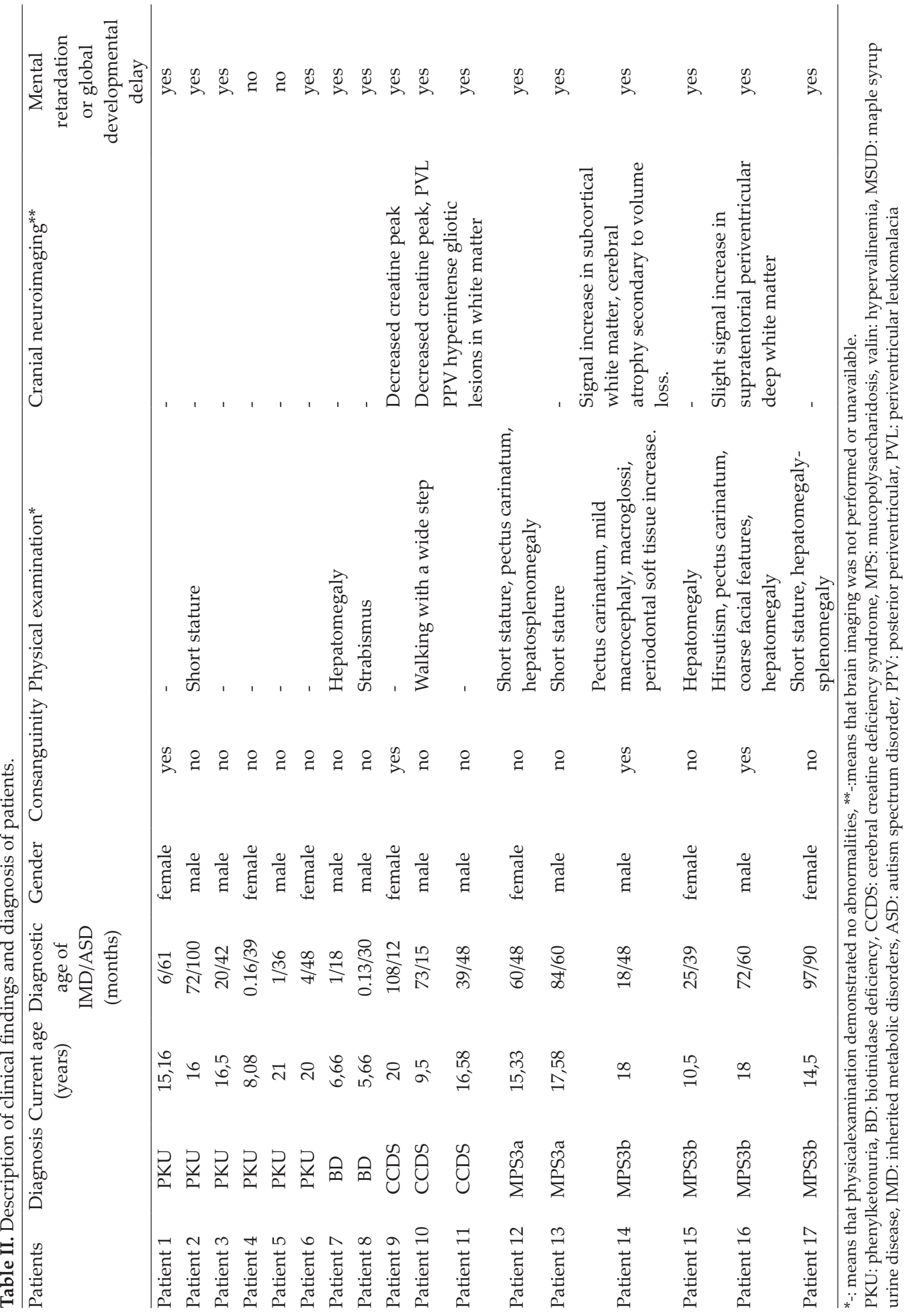




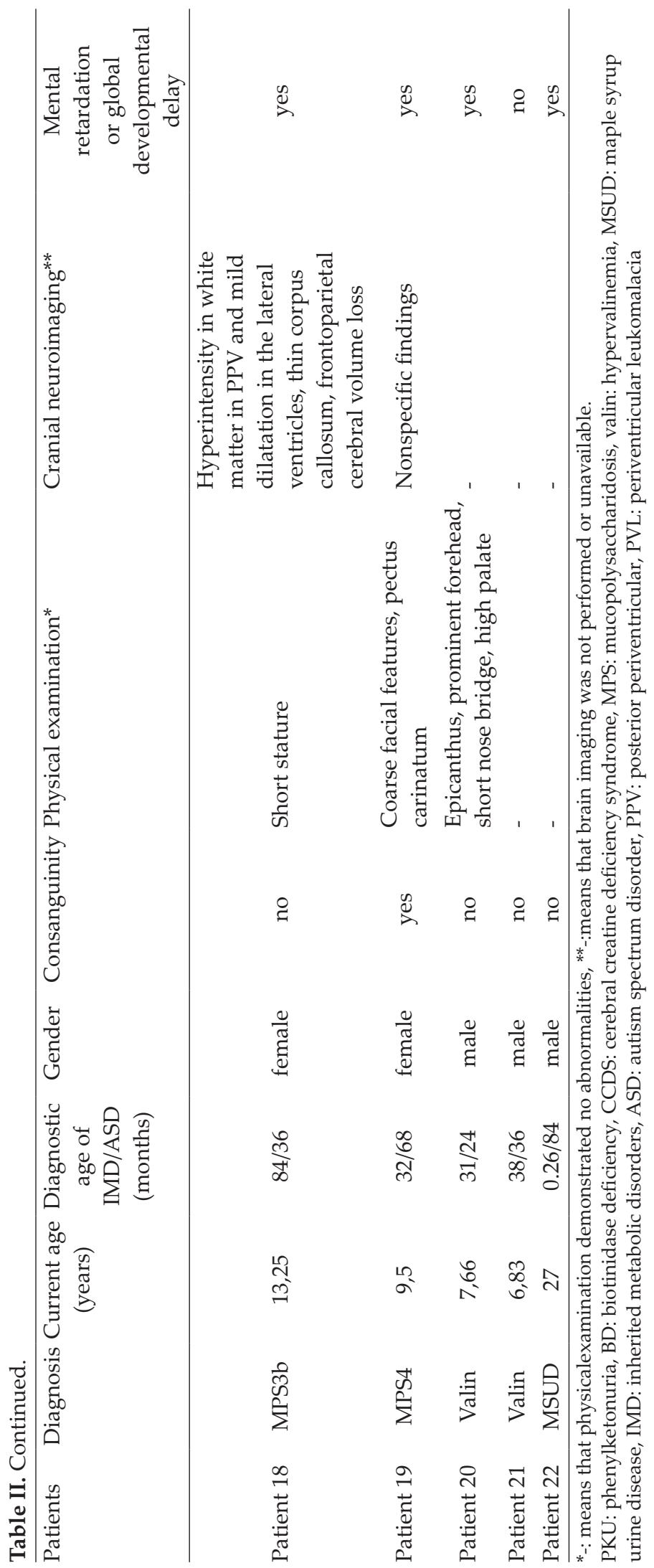


the most common of the seven MPS with a heterogeneous presentation and a progressive clinical course. It is characterized by progressive neurocognitive decline, behavioral difficulties and relatively mild somatic manifestations. ${ }^{16}$ Although the incidence of the subtypes varies according to geographic distribution Type-A and Type-B are more common than Type-C and Type-D. ${ }^{16,17}$ Since MPS 3 is characterized by early-onset developmental delay and subtle somatic features in early years, the important point here is that young children with MPS 3 are easily misdiagnosed with idiopathic developmental delay, attention deficit hyperactivity disorder or ASD as were the cases in our study. The diagnoses of MPS Type $3 a$ and Type $3 b$ were given after the diagnosis of ASD with an 18 and 24 months delay, respectively. Therefore, children with any developmental delay, especially with a characteristic somatic feature or behavioral abnormality, should be screened for MPS $3 .{ }^{16}$ Otherwise these children who are misdiagnosed may be referred to more invasive testing, dietary restrictions or even unproven alternative therapies. ${ }^{18}$

We also had one case with MPS-4 (Morquio A Syndrome) and ASD. To the best of our knowledge, the association between Morquio A Syndrome and autism has never been published before. It is caused by impaired catabolism of specific glycosaminoglycans (GAGs) keratan sulfate (KS) and chondroitin -6- sulfate (C6S). The accumulation of these GAGs becomes evident primarily in the cartilage and causes multi-systemic impairments. ${ }^{19-21}$ It is widely accepted that the syndrome is not directly related to the central nervous system (CNS). However a recent study has speculated that the biochemical mechanism, a kind of interplay between mitochondrial, calcium and lysosomal trafficking dysfunction may contribute to potential CNS involvement. Behavioral problems have been previously reported in MPS 4 and only one patient with ASD has been briefly mentioned in the same study. Although the evidence is still insufficient, the physiological role to coordinate the formation of neuroaxonal connections of KS and C6S in CNS is suggested in the early phases of brain development. ${ }^{22}$ Therefore it is plausible that we have defined a case with MPS 4 and a definite diagnosis of ASD. Nevertheless, MPS 4A and ASD coexistence may be coincidental.

We had two cases with partial Biotinidase Deficiency and ASD diagnosis. Biotinidase Deficiency is a metabolic disorder with homozygous or compound heterozygous variants. ${ }^{23}$ In the literature there has been only one case reported with partial biotinidase deficiency and ASD. The case was almost 4 years of age when he received the diagnosis and after beginning cofactor biotin treatment his autistic behavioral patterns were not resolved. It has been suggested that serious neurological problems such as autistic behaviors could be irreversible even with biotin supplementation. ${ }^{24}$ There should be further studies to explore the underlying mechanism relating to neurodevelopmental impairment in these cases because our two cases received their diagnosis in their first months and began biotin therapy early, however, they showed autistic features later at approximately two years of age. Coexistence of partial biotinidase deficiency, ASD and seizures is very likely coincidental in early diagnosed patients.

We had three cases with CCDS and ASD diagnosis. Loss-of-function mutations in the creatine transporter which transports creatine at the blood-brain barrier and into the neurons causes CCDS. Patients with these syndromes show various neurodevelopmental disorders, including developmental delay, seizures and intellectual disability; many of which are also evident in children with ASD. ${ }^{25}$ The observation of autistic symptoms in patients with CCDS implies that impairment in creatine metabolism may play a role in the neurobiology of ASD. Therefore, it may represent a treatable cause of ASD. ${ }^{26}$ Patients with all forms of CCDS have been reported to have ASD symptoms. ${ }^{26,27}$ Although Schulze et al. $^{26}$ has revealed a very low prevalence of CCDS in children with nonsyndromic ASD and no obvious association 
between creatine metabolites and autism, we had three cases with this syndrome and, two of them received the diagnoses of CCDS after the diagnosis of ASD.

We had one case with a diagnosis of MSUD and ASD.Branched-chain $\alpha$-ketoacid dehydrogenase catalyzes the critical step in the branched-chain amino acid catabolic pathway and mutations in the complex disrupt many fundamental metabolic pathways and cause multiple human diseases including MSUD, autism, and other related neurological disorders. ${ }^{28}$ Maple syrup urine disease is a disorder of branched-chain keto acid metabolism. In previous literature three children have been reported with an intermediate form of MSUD characterized by intellectual disability, seizures, autistic features, and movement disorder. ${ }^{29}$ The patient in the study demonstrated intellectual disability and autistic features with no abnormalities on his physical examination.

There are two cases with hypervalinemia in our study. They were identified during the evaluation of their developmental delay and autistic features. Very few cases of hypervalinemia have been previously reported in literature. Swarna et al. ${ }^{30}$ have identified two cases of hypervalinemia characterized by distinct physical and intellectual disability and muscular atrophy. However, our two patients had language, social-emotional delay and mild cognitive delay with normal physical growth.

The limitation of this study was that data relating to the diagnosis of ASD was obtained from their medical records and there was no standard documentation relating to the progress of ASD. This is because they were being followed up for ASD at different health centers around Turkey. Additionally, other well-known metabolic disorders with autistic features such as classical homocystinuria, urea cycle disorders, and purine metabolic pathway disorders were not presented in the study as possible cases with these disorders did not have a definitive diagnosis of ASD during the study period.
The main strength of the study is its' unique ability to address the co-occurrence of ASD in a large sample including rare metabolic diagnoses such as MPS Type $3 b$ and Type 4 and Hypervalinemia. Furthermore, the time of both IMD and ASD diagnosis and the clinical progress of their metabolic disorders were addressed to underline the treatable conditions with the aim of contributing to the literature.

In conclusion, we have presented some inherited metabolic disorders associated with ASD. Phenylketonuria and Mucopolysaccharidosis were the most common diagnoses in our study. The diagnoses of CCDS and MPS Type 3 were later than the diagnosis of ASD. In addition, rare entities such as MPS Type $3 \mathrm{~b}$ and Type 4 and Hypervalinemia were also reported to be associated with autism. Viewing the whole picture with this awareness and implementing intervention strategies considering the $\mathrm{CO}^{-}$ occurrence of both disorders should be an essential component of any treatment.

\section{Author contribution}

The authors confirm contribution to the paper as follows: study conception and design: TÇY, TC; data collection: TÇY, BBG, HTA; analysis and interpretation of results: TÇY, BBG, ENÖ, TC; draft manuscript preparation: TÇY, BBG, HTA, ENÖ. All authors reviewed the results and approved the final version of the manuscript.

\section{Ethical approval}

The study protocol was approved by the Ethics Committee of Hacettepe University Medical Faculty (GO 18/407) and the participation involved informed consent.

\section{Source of funding}

There is no any financial source.

\section{Conflict of interest}

The authors declare no conflict of interest. 


\section{REFERENCES}

1. Lappe M, Lau L, Dudovitz RN, Nelson BB, Karp EA, Kuo AA. The diagnostic odyssey of autism spectrum disorder. Pediatrics 2018; 141(Suppl 4): S272-S279.

2. Ghaziuddin M, Al-Owain M. Autism spectrum disorders and inborn errors of metabolism: an update. Pediatr Neurol 2013; 49: 232-236.

3. Simons A, Eyskens F, Glazemakers I, van West D. Can psychiatric childhood disorders be due to inborn errors of metabolism? Eur Child Adolesc Psychiatry 2017; 26: 143-154.

4. Sedel F, Baumann N, Turpin JC, Lyon-Caen O, Saudubray JM, Cohen D. Psychiatric manifestations revealing inborn errors of metabolism in adolescents and adults. J Inherit Metab Dis 2007; 30: 631-641.

5. Wertheim-Tysarowska K, Gos M, Sykut-Cegielska J, Bal J. Genetic analysis in inherited metabolic disorders--from diagnosis to treatment. Own experience, current state of knowledge and perspectives. Dev Period Med 2015; 19: 413-431.

6. Al-Owain M, Al-Zaidan H, Al-Hassnan Z. Map of autosomal recessive genetic disorders in Saudi Arabia: concepts and future directions. Am J Med Genet A 2012; 158A: 2629-2640.

7. Schiff M, Benoist JF, Aissaoui S, et al. Should metabolic diseases be systematically screened in nonsyndromic autism spectrum disorders? PLoS One 2011; 6: e21932.

8. Kiykim E, Zeybek CA, Zubarioglu T, et al. Inherited metabolic disorders in Turkish patients with autism spectrum disorders. Autism Res 2016; 9: 217-223.

9. Zablotsky B, Colpe LJ, Pringle BA, Kogan MD, Rice C, Blumberg SJ. Age of parental concern, diagnosis, and service initiation among children with autism spectrum disorder. Am J Intellect Dev Disabil 2017; 122: 49-61.

10. Maenner MJ, Schieve LA, Rice CE, et al. Frequency and pattern of documented diagnostic features and the age of autism identification. J Am Acad Child Adolesc Psychiatry 2013; 52: 401-413.e8.

11. Shattuck PT, Durkin M, Maenner M, et al. Timing of identification among children with an autism spectrum disorder: findings from a populationbased surveillance study. J Am Acad Child Adolesc Psychiatry 2009; 48: 474-483.

12. De Jaco A, Mango D, De Angelis F, et al. Unbalance between excitation and inhibition in phenylketonuria, a genetic metabolic disease associated with autism. Int J Mol Sci 2017; 18: 941-951.
13. Mazlum B, Anlar B, Kalkanoglu-Sivri HS, KarliOguz K, Ozusta S, Unal F. A late-diagnosed phenylketonuria case presenting with autism spectrum disorder in early childhood. Turk J Pediatr 2016; 58: 318-322.

14. Anderson PJ, Wood SJ, Francis DE, et al. Neuropsychological functioning in children with early-treated phenylketonuria: impact of white matter abnormalities. Dev Med Child Neurol 2004; 46: 230-238.

15. Christ SE, Moffitt AJ, Peck D. Disruption of prefrontal function and connectivity in individuals with phenylketonuria. Mol Genet Metab 2010; 99(Suppl 1): S33-S40.

16. Wijburg FA, Wegrzyn G, Burton BK, TylkiSzymanska A. Mucopolysaccharidosis type III (Sanfilippo syndrome) and misdiagnosis of idiopathic developmental delay, attention deficit/ hyperactivity disorder or autism spectrum disorder. Acta Paediatr 2013; 102: 462-470.

17. Valstar MJ, Ruijter GJ, van Diggelen OP, Poorthuis BJ, Wijburg FA. Sanfilippo syndrome: a mini-review. J Inherit Metab Dis 2008; 31: 240-252.

18. de Ruijter J, Valstar MJ, Wijburg FA Mucopolysaccharidosis type III (Sanfilippo syndrome): emerging treatment strategies. Curr Pharm Biotechnol 2011; 12: 923-930.

19. Khan S, Almeciga-Diaz CJ, Sawamoto K, et al. Mucopolysaccharidosis IVA and glycosaminoglycans. Mol Genet Metab 2017; 120: 78-95.

20. Tomatsu S, Yasuda E, Patel P, et al. Morquio A syndrome: diagnosis and current and future therapies. Pediatr Endocrinol Rev 2014; 12(Suppl 1): 141-151.

21. Harmatz P, Mengel KE, Giugliani R, et al. The Morquio A Clinical Assessment Program: baseline results illustrating progressive, multisystemic clinical impairments in Morquio A subjects. Mol Genet Metab 2013; 109: 54-61.

22. Davison JE, Kearney S, Horton J, Foster K, Peet AC, Hendriksz CJ. Intellectual and neurological functioning in Morquio syndrome (MPS IVa). J Inherit Metab Dis 2013; 36: 323-328.

23. Kury S, Ramaekers V, Bezieau S, Wolf B. Clinical utility gene card for: biotinidase deficiency-update 2015. Eur J Hum Genet 2016; 24. doi: 10.1038/ ejhg.2015.246.

24. Zaffanello M, Zamboni G, Fontana E, Zoccante L, Tato L. A case of partial biotinidase deficiency associated with autism. Child Neuropsychol 2003; 9: 184-188. 
25. Uemura $\mathrm{T}$, Ito $\mathrm{S}$, Ohta $\mathrm{Y}$, et al. Abnormal N-glycosylation of a novel missense creatine transporter mutant, G561R, associated with cerebral creatine deficiency syndromes alters transporter activity and localization. Biol Pharm Bull 2017; 40: 49-55.

26. Schulze A, Bauman M, Tsai AC, et al. Prevalence of creatine deficiency syndromes in children with nonsyndromic autism. Pediatrics 2016; 137: e20152672.

27. Schulze A. Creatine deficiency syndromes. Handb Clin Neurol 2013; 113: 1837-1843.
28. Jia F, Cui M, Than MT, Han M. Developmental defects of caenorhabditis elegans lacking branchedchain $\alpha$-ketoacid dehydrogenase are mainly caused by monomethyl branched-chain fatty acid deficiency. J Biol Chem 2016; 291: 2967-2973.

29. Bindu PS, Shehanaz KE, Christopher R, Pal PK, Ravishankar S. Intermediate maple syrup urine disease: neuroimaging observations in 3 patients from South India. J Child Neurol 2007; 22: 911-913.

30. Swarna M, Jyothy A, Usha Rani P, Reddy PP. Amino acid disorders in mental retardation: a two-decade study from Andhra Pradesh. Biochem Genet 2004; 42: 85-98. 